\title{
Different effects of granulocyte colony- stimulating factor and erythropoietin on erythropoiesis
}

\author{
Tzu-Lin Chen ${ }^{1 \dagger}$, Ya-Wen Chiang ${ }^{1 \dagger}$, Guan-Ling Lin², Hsin-Hou Chang ${ }^{1,2}$, Te-Sheng Lien ${ }^{1}$, Min-Hua Sheh ${ }^{1}$ \\ and Der-Shan Sun ${ }^{1,2^{*}}$ (D)
}

\begin{abstract}
Background: Red blood cells are the most abundant cells in the blood that deliver oxygen to the whole body. Erythropoietin (EPO), a positive regulator of erythropoiesis, is currently the major treatment for chronic anemia. Granulocyte colony-stimulating factor (G-CSF) is a multifunctional cytokine and a well-known regulator of hematopoietic stem cell proliferation, differentiation, and mobilization. The use of EPO in combination with G-CSF has been reported to synergistically improve erythroid responses in a group of patients with myelodysplastic syndromes who did not respond to EPO treatment alone; however, the mechanism remains unclear.

Methods: C57BL/6 J mice injected with G-CSF or EPO were used to compare the erythropoiesis status and the efficiency of erythroid mobilization by flow cytometry.

Results: In this study, we found that G-CSF induced more orthochromatophilic erythroblast production than did EPO in the bone marrow and spleen. In addition, in contrast to EPO treatments, G-CSF treatments enhanced the efficiency of the mobilization of newly synthesized reticulocytes into peripheral blood. Our results demonstrated that the effects of G-CSF on erythropoiesis and erythrocytic mobilization were independent of EPO secretion and, in contrast to EPO, G-CSF promoted progression of erythropoiesis through transition of early stage R2 (basophilic erythroblasts) to late stage R4 (orthochromatophilic erythroblasts).

Conclusions: We demonstrate for the first time that G-CSF treatments induce a faster erythropoiesis-enhancing response than that of EPO. These findings suggest an alternative approach to treating acute anemia, especially when patients are experiencing a clinical emergency in remote areas without proper blood bank supplies.
\end{abstract}

Keywords: Granulocyte colony-stimulating factor, Erythropoietin, Erythropoiesis, Reticulocyte, Erythrocytic mobilization

\section{Background}

Red blood cells (RBCs) are the most abundant cells in the blood and are essential for oxygen transport around the body. After birth, the site of erythropoiesis switches from the fetal liver to the bone marrow (BM) and spleen. In humans, the $B M$ is the major site for steady-state erythropoiesis. In contrast, in mice, in addition to the $\mathrm{BM}$, the spleen plays a minor role $(10 \%)$ in steady-state erythropoiesis $[1,2]$. Under stressful conditions, such as

\footnotetext{
* Correspondence: dssun@mail.tcu.edu.tw

${ }^{\dagger}$ Equal contributors

'Department of Molecular Biology and Human Genetics, Tzu-Chi University, No. 701, Section 3, Zhong-Yang Road, Hualien 97004, Taiwan, Republic of China

${ }^{2}$ Institute of Medical Sciences, Tzu-Chi University, Hualien, Taiwan
}

bleeding or acute anemia, the spleen in humans and mice plays a major role in stress erythropoiesis $[3,4]$. Hematopoietic stem cells (HSCs) reside in BM niches where cytokines or signals generated by stromal cells consisting of endothelial cells, osteoblasts, and macrophages regulate the differentiation of various blood lineage, including erythroid cells [5]. HSCs first differentiate into megakaryocyte-erythroid progenitors, subsequently into the burst-forming unit-erythroids (BFU-Es), and finally into the colony-forming unit-erythroids (CFU-Es). CFU-Es are more mature than BFU-Es and appear earlier, namely at 2-3 days in mice and 5-8 days in humans, compared with 5-8 days in mice and 10-14 days in humans for BFU-Es. In addition, CFU-Es form

(c) The Author(s). 2018 Open Access This article is distributed under the terms of the Creative Commons Attribution 4.0 International License (http://creativecommons.org/licenses/by/4.0/), which permits unrestricted use, distribution, and 
smaller colonies than do BFU-Es when cultured in methylcellulose [2, 3]. Erythropoietin (EPO), a 30.4-kDa glycoprotein mainly synthesized by the kidneys, is the main regulator of erythroid cell proliferation, differentiation, and survival [6]. EPO production is upregulated under hypoxic conditions through the activity of the hypoxia-inducible transcription factor (HIF) [7, 8]. The EPO receptor (EPOR) is expressed dominantly on CFUEs and gradually downregulated during erythroid differentiation $[9,10]$. Upon stimulation initiated by EPO binding to the EPORs, CFU-Es develops into proerythroblasts, subsequently into basophilic erythroblasts, then polychromatic erythroblasts, and finally orthrochromatic erythroblasts. The final stage of erythroid differentiation involves the enucleation and maturation of reticulocytes into circulating erythrocytes [11].

Anemia can develop from loss of RBCs, a reduction in $\mathrm{RBC}$ production, increased destruction of RBCs, or a shorter RBC lifespan. The World Health Organization defines anemia as a hemoglobin level lower than $12 \mathrm{~g} / \mathrm{dl}$ in women and lower than $13 \mathrm{~g} / \mathrm{dl}$ in men [12]. Except for patients with inherited hematopoietic disease, the highest rates of anemia are observed in patients with chronic diseases such as those of the kidney and heart, cancer, inflammatory bowel disease, rheumatoid arthritis, and human immunodeficiency virus (HIV) [13]. Recombinant human EPO (rhEPO) has been used medically for more than 20 years and has generated a multibillion dollar market annually. However, since 1998, some severe adverse effects such as EPO-associated pure red cell aplasia by long-term EPO injection induced neutralizing antibodies have been reported [14, 15]. Other side effects such as hypertension, increased risk of venous thromboembolism, stroke, and death have also been reported [16]. Currently available erythropoiesis-stimulating agents (ESAs) are variations of EPO generated by human cells or Chinese hamster ovary cells. New ESAs (e.g., peptide-based erythropoietic agents), activation of endogenous EPO production through HIF stabilization and GATA1 inhibition, and EPO gene therapy have been developed; however, none of these new methods have shown efficacy superior to that of existing ESAs [17]. Taken together, the development of new anemia therapies with satisfactory levels of efficacy and safety and faster action is required.

Our previous study found that granulocyte colonystimulating factor (G-CSF) could mobilize newly synthesized erythrocytes to the peripheral blood (PB) and promote erythrocytic differentiation and proliferation in vitro and ex vivo [18]. The present study further investigated the differences between G-CSF and EPO on erythropoiesis. After mice had been treated with G-CSF and EPO, the erythropoiesis status in the BM and spleen were compared using flow cytometry. The stimulation of early erythroid progenitor subsets and temporal regulation of erythroid progenitor mobilization were characterized. The mechanism of G-CSF promotion of erythropoiesis was also discussed.

\section{Methods}

Toxins and mice

B. anthracis lethal toxin (LT) was provided by the Institute of Preventive Medicine, National Defense Medical Center (Taipei, Taiwan), and purified as previously described [19]. EGFP mice [C57BL/6 J-Tg (Pgk1-EGFP) 03Narl] provided by Professor Chou CK [20] and C57BL/6 J mice were purchased from the National Laboratory Animal Center (Taipei, Taiwan). Animals were maintained in a specific pathogen-free environment in the experimental animal center of Tzu Chi University (Hualien, Taiwan).

\section{Analysis of erythropoiesis status in the BM and spleen} C57BL/6 J mice (male, 10-12 weeks old) were retroorbitally injected with $2 \mathrm{IU} / \mathrm{g}$ rhEPO (Neorecormon', Roche, Mannheim, Germany) or $55 \mu \mathrm{g} / \mathrm{kg}$ recombinant human G-CSF (Filgrastim, Kirin, Tokyo) once daily on 3 consecutive days. Animals treated with an identical volume of saline served as negative controls. BM cells were isolated as previously described [21]. Spleens were minced using the plunger of a 50-ml syringe and resuspended using a P1000 Pipetman (Gilson, Middleton, WI, USA) to create a singlecell suspension. Cells were blocked with RPMI-1640 medium (Gibco Laboratories) containing 5\% bovine serum albumin at $37{ }^{\circ} \mathrm{C}$ for $1 \mathrm{~h}$ and subsequently incubated with rat anti-mouse fluorescein isothiocyanate-conjugated CD71 antibody (BioLegend) and rat antimouse allophycocyanin (APC)-conjugated TER-119 antibody (BD Immunocytometry System) at $37^{\circ} \mathrm{C}$ for $1 \mathrm{~h}$. After washing with phosphatebuffered saline (PBS), the cells were resuspended in $1 \mathrm{ml}$ of PBS and analyzed using a Beckman Coulter Gallios ${ }^{\mathrm{Tm}}$ flow cytometer (Beckman Coulter, CA, USA).

\section{Flow cytometry analysis of mobilized erythrocytes in EGFP mice}

Enhanced green fluorescent protein (EGFP) mice (male, 8 months old) were injected with $55 \mu \mathrm{g} / \mathrm{kg}$ G-CSF or $2 \mathrm{IU} / \mathrm{g}$ EPO retro-orbitally once daily on 3 consecutive days. PB was collected retro-orbitally at 20,40 , and $60 \mathrm{~h}$ after initial injection. Cells were incubated with rat antimouse APC-conjugated TER-119 antibody at $37{ }^{\circ} \mathrm{C}$ for $1 \mathrm{~h}$ and subsequently with $5 \mu \mathrm{M}$ of RNA-selective dye F22 [22] at $37{ }^{\circ} \mathrm{C}$ for $30 \mathrm{~min}$. After washing with PBS, the cells were analyzed using a Beckman Coulter Gallios $^{\text {Tix }}$ flow cytometer.

\section{EPO and soluble P-selectin immunoassay}

C57BL/6 J mice (male, 10-12 weeks old) were treated with $55 \mu \mathrm{g} / \mathrm{kg}$ G-CSF administered by retro-orbital injection once daily on 2 consecutive days for the EPO immunoassay or 5 consecutive days for the soluble P-selectin 
immunoassay. Animals treated with an identical volume of saline served as negative controls. C57BL/6 J mice that were retro-orbitally injected with $1.5 \mathrm{mg} / \mathrm{kg} \mathrm{LT}$ and untreated served as positive controls for the EPO immunoassay and the soluble P-selectin immunoassay, respectively. PB (serum for the EPO immunoassay and plasma for the soluble Pselectin immunoassay) was collected retro-orbitally at 22 , 44 , and $66 \mathrm{~h}$ after initial injection for the EPO immunoassay, and day 0 before initial injection and days 1-5 after initial injection for the soluble P-selectin immunoassay. The EPO and soluble P-selectin immunoassays were performed using an enzyme-linked immunosorbent assay (ELISA) in accordance with the manufacturers' instructions (Quantikine $^{\bullet}$ Mouse/Rat EPO immunoassay, R\&D systems; Mouse P-selectin ELISA kit, RayBiotech, Inc., USA).

\section{Hematopoietic parameter detection}

C57BL/6 J mice (male, 10-12 weeks old) were treated with $55 \mu \mathrm{g} / \mathrm{kg}$ G-CSF or $1 \mathrm{mg} / \mathrm{kg}$ recombinant mouse Pselectin (purified mouse P-selectin-IgG fusion protein; $\mathrm{BD}$ Pharmingen) once daily on 2 consecutive days through retro-orbital injection. The hematopoietic parameters were measured on day 2 after initial injection using an automated hematology analyzer (XP-300, Sysmex Corporation).

\section{Statistics}

All quantifiable data are presented as mean \pm standard deviation (SD). Statistical analysis was conducted by one-way analysis of variance (ANOVA) followed by the post-hoc Bonferroni-corrected $t$ test using the SPSS software, version 17.0 (SPSS Inc., Chicago, IL, USA). $P$ values lower than 0.05 indicated significant differences.

\section{Results}

\section{G-CSF enhanced R4 erythroblast cell production to a} greater extent than EPO did in the BM and spleen

To compare the erythropoiesis status after G-CSF and EPO treatments, C57BL/6 J mice were retro-orbitally injected with G-CSF or EPO once daily for 3 consecutive days. Erythropoiesis progression was analyzed in the BM (Fig. 1a) and spleen (Fig. 2a) through flow cytometry. Using antibodies against the erythroid markers CD71 and TER119, erythroblasts were divided into the following four populations from early to late sequential differentiation stages: proerythroblasts (R1, CD71 ${ }^{\text {high }} / \mathrm{TER}-119^{\text {med }}$ ), basophilic erythroblasts (R2, CD71 $1_{\text {high }} /$ TER- $119^{\text {high }}$ ), late basophilic and polychromatophilic erythroblasts (R3, CD71 ${ }^{\text {med }} /$ TER-119 $\left.9^{\text {high }}\right)$, and orthochromatophilic erythroblasts (R4, CD71 $1^{\text {low or }-/ T E R-119 ~}{ }^{\text {high }}$ ) (Figs. 1b and $2 b)[18,21,23]$. In agreement with the findings of other studies, in mouse BM, G-CSF enhanced more nonerythroid cell populations (NE, TER-119-) [24] than EPO did (Fig. 1c). In contrast, EPO substantially increased the total number of erythroid cells [6] (Fig. 1d). Although total erythroid cell counts in the BM of mice from the G-CSFtreated groups were lower than those of mice from the EPO-treated groups, G-CSF elicited a markedly larger R4 population than EPO did (Fig. 1e).

The spleen is a secondary organ for erythropoiesis but not for granulopoiesis $[2-4,25]$. In characterizing the differentiation progression in the mouse spleen, we found that G-CSF treatments did not increase the nonerythroid cell population or total erythroid cell number (Fig. 2c, d). Compared with the responses in the BM, EPO upregulated the percentage of early erythroblasts (R1 and R2) and downregulated the late stages of erythroblasts (R3 and R4) (Fig. 2e), whereas G-CSF reduced the percentage of early R1, R2, and R3 cells and considerably increased the number of late-stage R4 erythroblasts (Fig. 2e). These results collectively suggest that G-CSF enhances late-stage $\mathrm{R} 4$ erythroblast cell production to a greater extent than $\mathrm{EPO}$ does in the BM and spleens of mice.

\section{G-CSF mobilized more newly synthesized reticulocytes than EPO did}

Using an EGFP transgenic mouse model, we found that G-CSF caused newly synthesized erythrocytes to mobilize to the $\mathrm{PB}$ and induced erythrocytes to mobilize into the PB faster than EPO [18]. After using the same experimental strategy (Fig. 3a), our data revealed that the mobilization efficiencies of newly synthesized erythrocytes (R1, EGFP ${ }^{\text {high }} /$ TER-119 ${ }^{\text {high }}$ ) were higher at $20 \mathrm{~h}$ under G-CSF treatment than under EPO, and were similar between G-CSF and EPO treatments at 40 and $60 \mathrm{~h}$ after initial injection (Fig. 3b, c). Because G-CSF promoted more R4 erythroblast synthesis than EPO did in the BM and spleen (Figs. 1 and 2), we hypothesized that G-CSF may promote reticulocyte mobilization to a greater extent than EPO. Because only reticulocytes exhibit residue ribonucleic acid (RNA) expression among newly synthesized erythrocyte populations [26], F22, a RNA-selective dye [22], combined with TER-119 was used to discriminate reticulocytes from matured erythrocytes in newly synthesized erythrocytes. Over two time courses, 20 and $40 \mathrm{~h}$, G-CSF preferred to mobilize newly synthesized reticulocytes (F22 ${ }^{\text {high }} /$ EGFP $^{\text {high }} /$ TER- $119^{\text {high }}$ ) to a greater extent than EPO did (Fig. 3b, d).

\section{G-CSF-dependent enhancement of erythropoiesis and RBC mobilization were not mediated by eliciting EPO and the P-selectin pathway}

The transcription factor HIF-1 $\alpha$ can be upregulated by GCSF and then binds to an EPO promoter to increase circulating EPO levels after five consecutive G-CSF injections [27]. Accordingly, we hypothesized that G-CSF may stimulate erythropoiesis and mobilize erythrocytes by increasing the synthesis of EPO. Our experimental design is shown in 
a

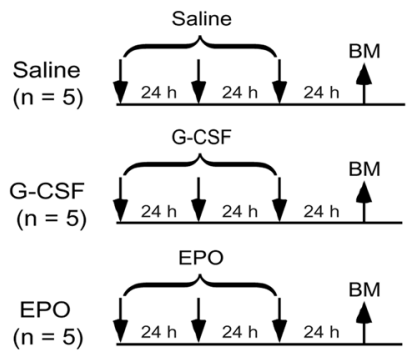

b
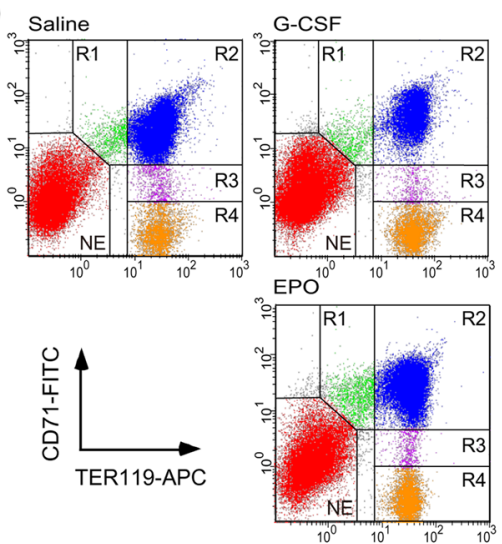

d

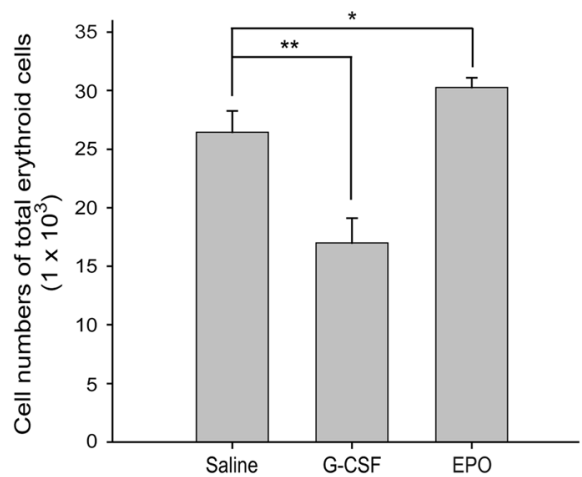

C

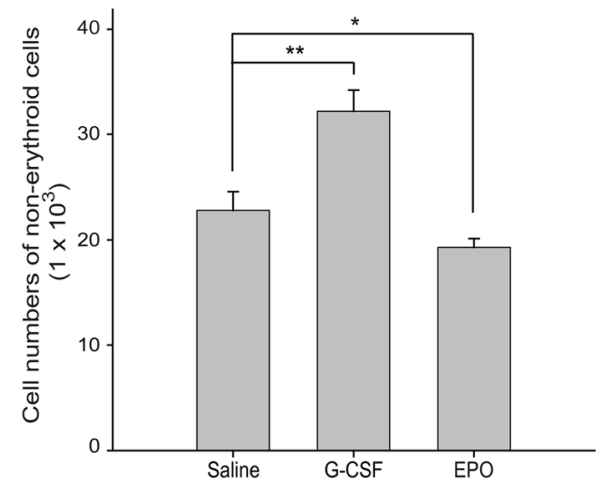

e

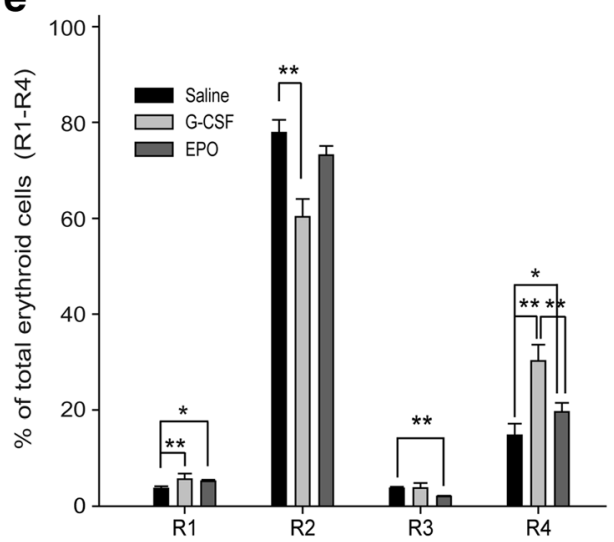

Fig. 1 Granulocyte colony-stimulating factor (G-CSF) treatment induced more R4 erythroblast cells than erythropoietin (EPO) treatment did in bone marrow (BM). a The experimental outline used to measure erythropoiesis status after G-CSF $(n=5)$ and EPO $(n=5)$ treatments in the BM is depicted. Saline-treated mice $(n=5)$ were used as negative controls. Flow cytometry analysis was performed $72 \mathrm{~h}$ after initial injection. The

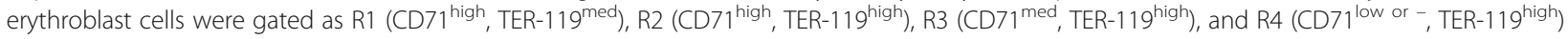
in all groups. Nonerythroid cells were gated as NE (TER-119 negative) (b). Cell numbers of nonerythroid (c) and total erythroid cells (sum of R1, $R 2, R 3$, and R4) (d), and the percentage of individual erythroblasts (R1, R2, R3, and R4) in the total number of erythroid cells in each group (e) were quantified. Data are reported as mean \pm SD. ${ }^{*} P<0.05,{ }^{*} P<0.01$

Fig. 4a. Treatment with Bacillus anthracis, a LT that can induce hypoxia-elicited EPO secretion in mice [28], was used as a positive control. In agreement with a previous study [28], our results revealed that circulating EPO levels gradually increased following LT administration in mice [28]. In contrast, circulating EPO levels were not upregulated at all examined time points after G-CSF treatment in mice (Fig. $4 \mathrm{~b}-\mathrm{d})$. These results suggest that the G-CSF-dependent enhancement of erythropoiesis and RBC mobilization are not mediated by eliciting EPO.
P-selectin is widely recognized as a cell adhesion receptor that mediates leukocyte rolling through binding to glycosylated moieties of the main ligand P-selectin glycoprotein ligand 1 (PSGL-1) [29]. The same property of P-selectin enables HSCs to adhere to stromal cells, especially vesicular endothelial cells, through P-selectin (HSC)-PSGL-1 (stroma) interactions [30]. G-CSF induced greater and faster mobilization of myeloid cells from BM in PSGL-1 knockout mice than in wild-type mice [31]. Studies have also suggested that G-CSF 


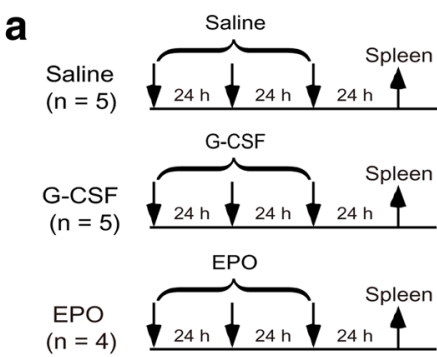

b
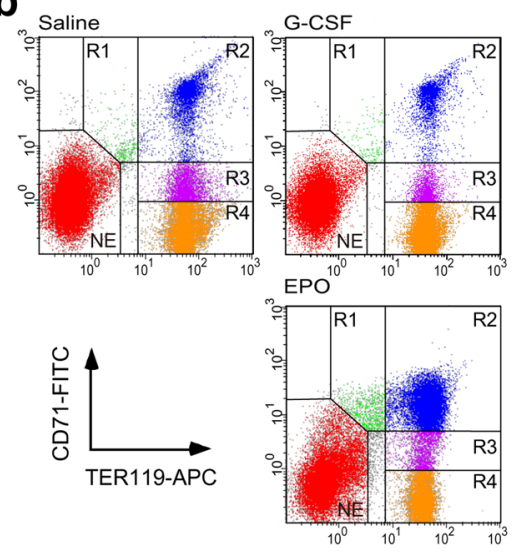

d

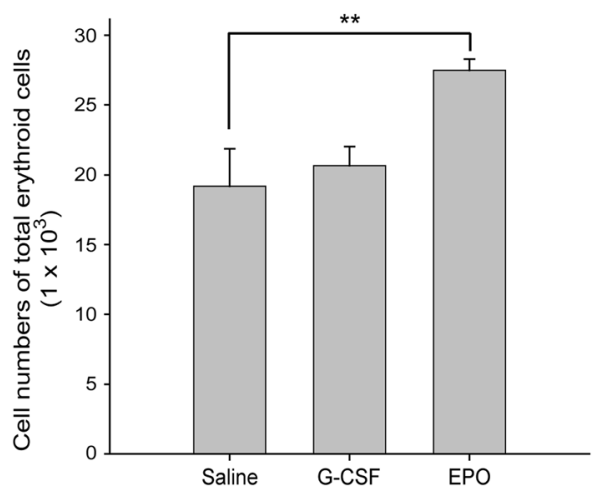

C

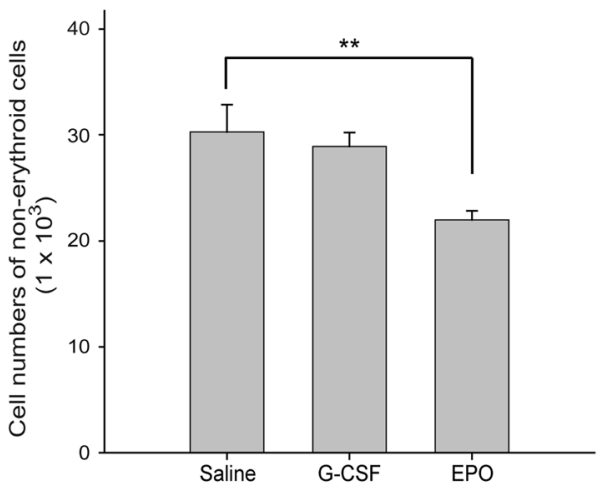

e

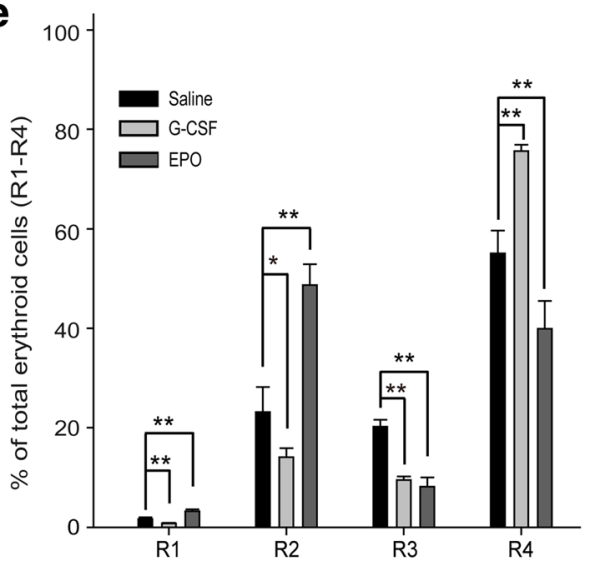

Fig. 2 Granulocyte colony-stimulating factor (G-CSF) treatment induced more R4 erythroblast cells than erythropoietin (EPO) treatment did in the spleen. a The experimental outline used to measure the erythropoiesis status after G-CSF $(n=5)$ and EPO $(n=4)$ treatments in the spleen is depicted. Saline-treated mice $(n=5)$ were used as negative controls. Flow cytometry analysis was performed $72 \mathrm{~h}$ after initial injection. The erythroblast cells were gated as R1, R2, R3, and R4 in all groups (see Fig. 1 for details). Nonerythroid cells were gated as NE (b). Cell numbers of nonerythroid (c) and total erythroid cells (sum of R1, R2, R3, and R4) (d), and the percentage of individual erythroblasts (R1, R2, R3, and R4) in the total number of erythroid cells in each group (e) were quantified. Data are reported as mean \pm SD. ${ }^{*} P<0.05$, ${ }^{*} P<0.01$

administration increases levels of circulating soluble Pselectin [32, 33]. This evidence collectively suggests that P-selectin/PSGL-1-mediated binding between erythroid precursors and stromal cells may partially control the mobilization and release of progenitor cells from BM into the blood stream, thereby indicating the possibility that G-CSF may enhance erythrocytic mobilization by eliciting circulating soluble P-selectin to compete with the P-selectin/PSGL-1-mediated precursor-stroma interaction. To reproduce G-CSF-mediated elicitation of circulating soluble P-selectin, G-CSF was injected into C57BL/6 J mice on 5 consecutive days (Fig. 5a). The levels of circulating soluble P-selectin increased significantly after G-CSF injection (Fig. 5b). Next, we designed an experiment to examine whether soluble P-selectin can increase the $\mathrm{RBC}$ counts in the PB (Fig. 5c). Although two doses of G-CSF increased the white blood cell (WBC) and RBC counts in the PB, the same effect was not observed when two equivalent doses of soluble P-selectin were applied (Fig. 5d, e). This finding suggests 
a

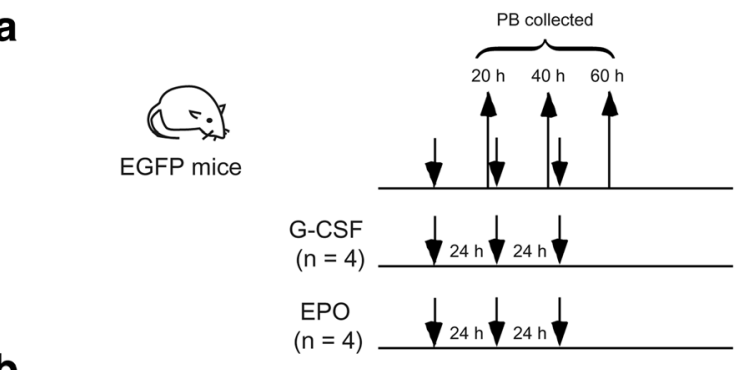

b
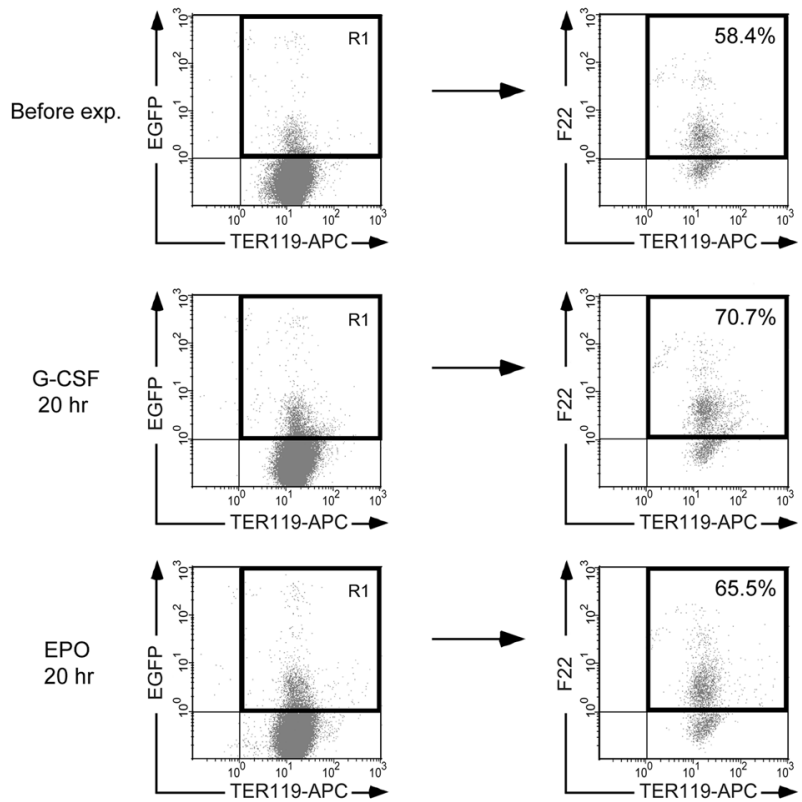

C

d
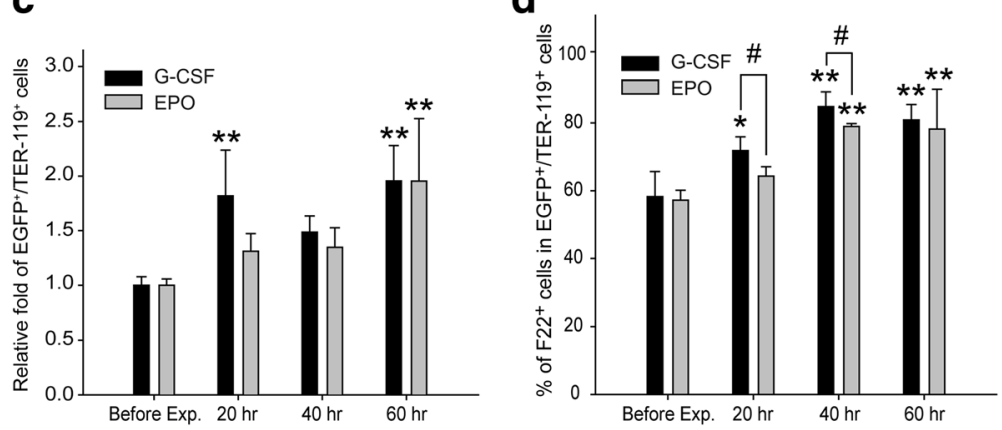

Fig. 3 Granulocyte colony-stimulating factor (G-CSF) treatment mobilized more newly synthesized reticulocytes than erythropoietin (EPO) treatment did. a The experimental outline for investigating erythrocyte mobilization in enhanced green fluorescent protein (EGFP) mice after G-CSF $(n=4)$ and EPO $(n=4)$ treatments is shown. Peripheral blood (PB) was collected 20,40, and $60 \mathrm{~h}$ after initial injection. Flow cytometry analysis was performed to analyze the relative fold of EGFP ${ }^{+} / T E R-119^{+}$cells (gated R1 region) compared with the before experiment (Before exp.) groups and the percentage of $\mathrm{F}_{2} 2^{+}$cells in $\mathrm{EGFP}^{+} / \mathrm{TER}-119^{+}$cells at $20 \mathrm{~h}$ (b). Quantitative results of the relative fold of EGFP $/ \mathrm{TER}^{+} 119^{+}$cells $(\mathbf{c})$ and the percentage of $\mathrm{F}_{2} 2^{+}$cells in EGFP ${ }^{+} / \mathrm{TER}-119^{+}$cells at 20,40 , and $60 \mathrm{~h}$ (d) are indicated. Data are reported as mean \pm SD. ${ }^{*} P<0.05$, * ${ }^{*} P<0.01$, versus the Before exp. groups; ${ }^{\#} P<0.05$

that G-CSF mobilization of erythrocytes into the PB is not primarily mediated through the P-selectin pathway.

\section{Discussion}

This study demonstrated that stimulation of erythropoiesis in mice with G-CSF is different to that with
EPO. Treatment with G-CSF elicited greater R4 erythroblast production than did treatment with EPO in the BM and spleen. Additionally, G-CSF induced higher mobilization levels of newly synthesized reticulocytes from the $\mathrm{BM}$ and spleen to the PB than EPO did. 
a

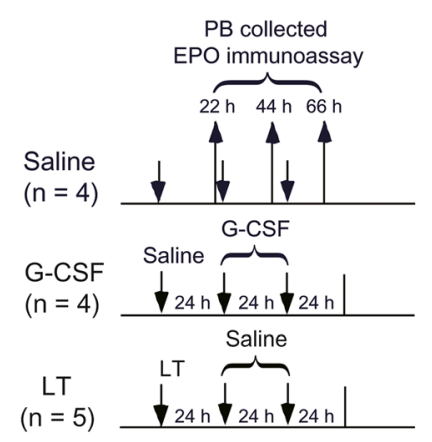

b

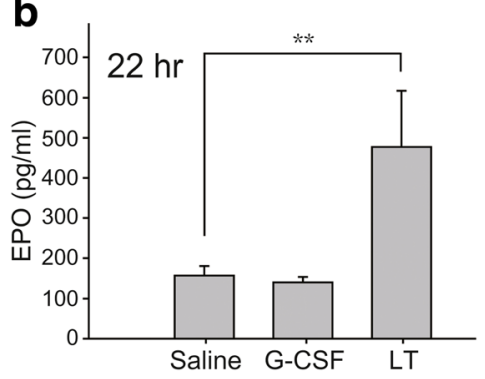

C

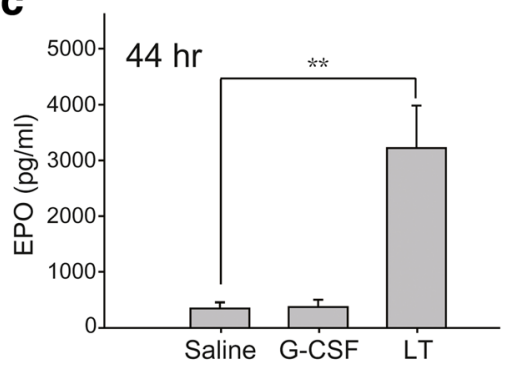

d

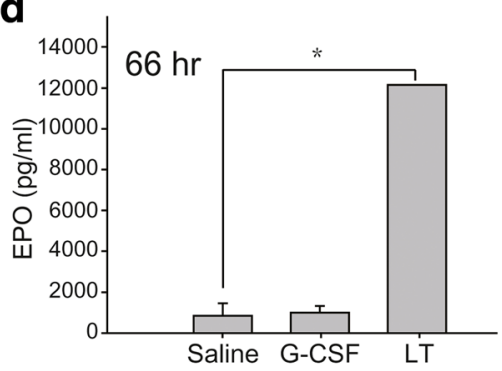

Fig. 4 Granulocyte colony-stimulating factor (G-CSF) treatment did not trigger erythropoietin (EPO) secretion. a The experimental outline used for the EPO immunoassay is illustrated. Mice were injected with G-CSF $(n=4)$ once daily after saline injection. EPO concentrations in the peripheral blood (PB) were detected by ELISA at $22 \mathrm{~h}(\mathbf{b}), 44 \mathrm{~h}(\mathbf{c})$, and $66 \mathrm{~h}$ (d) after initial injection. Saline $(n=4)$ and anthrax lethal toxin (LT) ( $n=5)$ treatments served as negative and positive controls, respectively. Data are reported as mean \pm SD. ${ }^{*} P<0.05,{ }^{* *} P<0.01$

G-CSF has been a US Food and Drug Administration (FDA)-approved drug for treating various hematopoietic defects for many years. In contrast to EPO, elicitation of neutralizing antibodies after G-CSF treatments has not been reported. Myelodysplastic syndromes (MDS) are a diverse group of diseases characterized by ineffective hematopoiesis and peripheral cytopenia with unknown mechanisms [34, 35]. As one of the disease hallmarks, approximately $85 \%$ of patients with MDS manifest anemia [36, 37]. Synergism between G-CSF and EPO treatments has ameliorated anemic conditions in groups of patients with MDS who did not respond to EPO treatments $[38,39]$. This suggests that the erythropoiesis-enhancing effect of the combination of GCSF and EPO has been empirically recognized by physicians and the medical community. Studies have shown that GCSF has antiapoptotic effects that protect erythroblasts from cell death [40-42]. However, the effects and mechanisms of G-CSF on erythropoiesis in vivo must be elucidated. Our results demonstrate that G-CSF could promote erythropoiesis by stimulating R4 erythroblast production and then mobilizing newly synthesized reticulocytes to a greater extent than EPO treatment. The effects partially agree with the findings of our previous study that small and medium progenitor cell colonies appeared earlier when tested with an erythroid colony-forming cell assay [18]. In contrast, treatments with G-CSF alone do not exhibit ameliorative effects on anemia in patients with MDS [42, 43]. In accordance with these findings, in our erythroid colony- forming cell experiment, G-CSF exhibited erythropoietic properties only when used with EPO [18]. This finding implied that G-CSF was unable to stimulate erythropoiesis without EPO. The EPOR is expressed dominantly on CFUEs and gradually downregulated during erythroid differentiation $[9,10]$. In addition, the G-CSF receptor is expressed on erythroid progenitors [42]. Because late-stage erythroblasts (R3 or R4) were upregulated after G-CSF treatment, synergism between G-CSF and EPO is likely accomplished by accelerating the erythropoietic process into the late stage. Recently, papers reported that G-CSF treatments enhanced hematopoietic stem and progenitor cell mobilization by enhancement of dipeptidylpeptidase 4 (CD26) activities and vascular permeability in the $\mathrm{BM}[44,45]$. This evidence may provide an additional explanation as to why G-CSF induced higher mobilization levels of newly synthesized reticulocytes from the $\mathrm{BM}$ and spleen to the $\mathrm{PB}$ than that of EPO. Although the responses that occur in $\mathrm{BM}$ with multilineage cell interactions are difficult to analyze, the mechanism warrants further investigation.

\section{Conclusions}

In conclusion, although G-CSF combined with EPO has been administered to treat anemia in patients with MDS, aplastic anemia, and HIV for many years [38, 39, 46, 47], the mechanism through which G-CSF promotes erythropoiesis remains unclear. This study demonstrates that GCSF promotes erythropoiesis independent of the secretion 
a
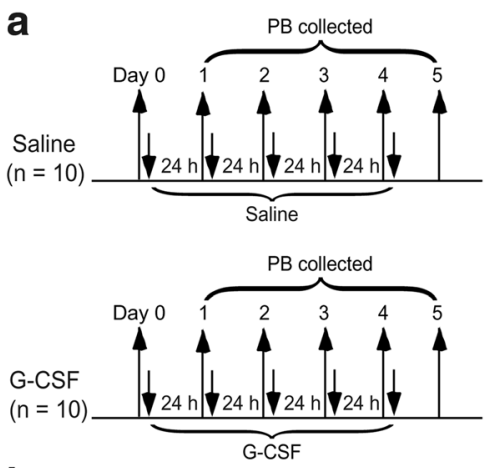

b

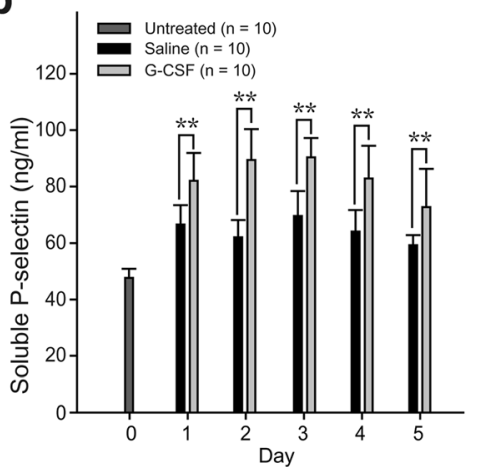

C $\{$ Untreated $(n=5)$,

G-CSF $(n=4)$ or P-selectin $(n=10)$ treated

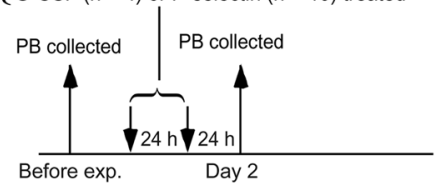

d

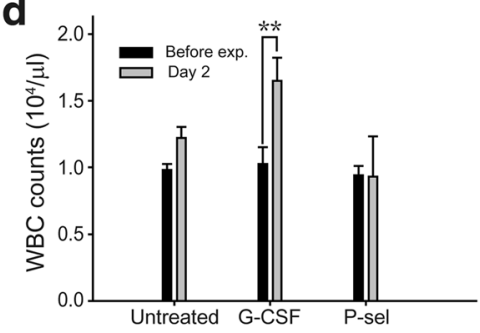

e

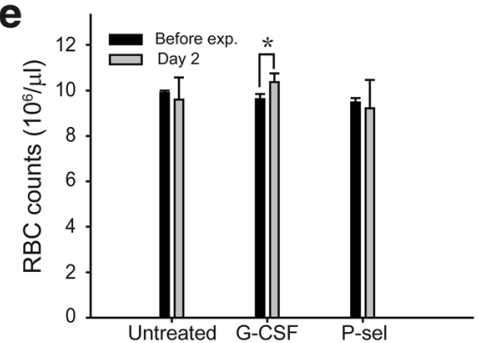

Fig. 5 Secretion of soluble P-selectin (P-sel) after granulocyte colony-stimulating factor (G-CSF) treatment was independent of erythrocytic mobilization. a The experimental outline to monitor the concentrations of soluble P-selectin after G-CSF treatment $(n=10)$ is illustrated. Untreated $(n=10)$ and saline-treated groups $(n=10)$ served as negative controls. The concentrations of soluble P-selectin were detected by ELISA (b). The experimental outline to detect the cell counts of white blood cells (WBCs) (d) and red blood cells (RBCs) (e) in PB after G-CSF $(n=4)$ and P-selectin $(n=10)$ treatments is shown $(\mathbf{c})$. Untreated groups $(n=5)$ served as negative controls. Data are reported as mean \pm \pm SD. ${ }^{*} P<0.05,{ }^{*} P<0.01$

of EPO and soluble P-selectin. In addition, G-CSF induces the production of levels of R4 erythroblasts to a greater extent than EPO does in the BM and spleen, and treatment with G-CSF mobilizes more newly synthesized reticulocytes to the PB than EPO does. These findings indicate an alternative method for ameliorating anemia, especially in situations where a patient is in immediate need of oxygen, such as those involving infectious diseases [18] or with clinical urgency in remote areas.

\section{Abbreviations}

APC: Allophycocyanin; BFU-E: Burst-forming unit-erythroid; BM: Bone marrow; CFU-E: Colony-forming unit-erythroid; EGFP: Enhanced green fluorescent protein; ELISA: Enzyme-linked immunosorbent assay; EPO: Erythropoietin; EPOR: Erythropoietin receptor; ESA: Erythropoiesis-stimulating agent; FDA: Food and Drug Administration; G-CSF: Granulocyte colony-stimulating factor; HIF: Hypoxia-inducible transcription factor; HIV: Human immunodeficiency virus; HSC: Hematopoietic stem cell; LT: lethal toxin; MDS: Myelodysplastic syndromes; PB: Peripheral blood; PBS: Phosphatebuffered saline; PSGL-1: P-selectin glycoprotein ligand 1; RBC: Red blood cell; rhEPO: Recombinant human erythropoietin; RNA: Ribonucleic acid;

SD: Standard deviation

\section{Acknowledgments}

We greatly appreciate Professor Chang YT (Department of Chemistry, National University of Singapore) for kindly providing the RNA-selective dye F22. We are grateful to Mr. Chen CC and the Department of Laboratory Medicine (Hualien Tzu Chi Medical Center) for their assistance with the complete blood count blood test. We thank Professor Shiue
CN (Department of Molecular Biology and Human Genetics, Tzu Chi University) for his valuable suggestions. We thank Professors Kau JH, Hsu HL, and Mr. Huang HH (Institute of Preventive Medicine, National Defense Medical Center) for generously providing anthrax LT. We also thank Professor Wang MH and his team (Experimental Animal Center, Tzu Chi University) for maintaining the experimental animals and pathogen-free environments. We acknowledge Wallace Academic Editing for editing this manuscript.

\section{Funding}

This work was supported by the Ministry of Science and Technology, Taiwan (MOST 103-2320-B-320-006).

\section{Availability of data and materials}

All data generated and/or analyzed during this study are available from the corresponding author upon reasonable request.

\section{Authors' contributions}

TLC, YWC, GLL, TSL, and MHS contributed by performing the experiments and analyzing the data. $\mathrm{HHC}$ designed the experiments and edited the manuscript. DSS designed the experiments, composed the main manuscript, and directed the study. All authors read and approved the final manuscript.

\section{Ethics approval}

The research protocols associated with the experimental mice were approved by the Institutional Animal Care and Use Committee of Tzu Chi University (approval no. 102087) and the National Defense Medical Center (approval no. AN-100-04).

\section{Competing interests}

The authors declare that they have no competing interests. 


\section{Publisher's Note}

Springer Nature remains neutral with regard to jurisdictional claims in published maps and institutional affiliations.

Received: 24 January 2018 Revised: 8 April 2018 Accepted: 16 April 2018 Published online: 02 May 2018

\section{References}

1. Vacha J, Hola J, Dungel J, et al. The distribution of erythropoiesis over the various anatomical regions of the erythropoietic system in some inbred strains of mice. Exp Hematol. 1982;10:768-73.

2. Halupa A, Bailey ML, Huang K, et al. A novel role for STAT1 in regulating murine erythropoiesis: deletion of STAT1 results in overall reduction of erythroid progenitors and alters their distribution. Blood. 2005;105:552-61.

3. Dzierzak E, Philipsen S. Erythropoiesis: development and differentiation. Cold Spring Harb Perspect Med. 2013;3:a011601.

4. Socolovsky M. Molecular insights into stress erythropoiesis. Curr Opin Hematol. 2007;14:215-24

5. Sugiyama T, Nagasawa T. Bone marrow niches for hematopoietic stem cells and immune cells. Inflamm Allergy Drug Targets. 2012:11:201-6.

6. Fisher JW. Erythropoietin: physiology and pharmacology update. Exp Bio Med (Maywood). 2003;228:1-14.

7. Schofield CJ, Ratcliffe PJ. Oxygen sensing by HIF hydroxylases. Nat Rev Mol Cell Biol. 2004:5:343-54.

8. Stockmann C, Fandrey J. Hypoxia-induced erythropoietin production: a paradigm for oxygen-regulated gene expression. Clin Exp Pharmacol Physiol. 2006;33:968-79.

9. Jelkmann W. Physiology and pharmacology of erythropoietin. Transfus Med Hemother. 2013:40:302-9.

10. Zhang J, Socolovsky M, Gross AW, et al. Role of Ras signaling in erythroid differentiation of mouse fetal liver cells: functional analysis by a flow cytometry-based novel culture system. Blood. 2003;102:3938-46.

11. Elliott S, Pham E, Macdougall IC. Erythropoietins: a common mechanism of action. Exp Hematol. 2008;36:1573-84

12. DeMaeyer $\mathrm{E}$, Adiels-Tegman M. The prevalence of anaemia in the world. World Health Stat Q. 1985;38:302-16.

13. Nissenson AR, Wade $S$, Goodnough $T$, et al. Economic burden of anemia in an insured population. J Manag Care Pharm. 2005;11:565-74.

14. Casadevall N, Nataf J, Viron B, et al. Pure red-cell aplasia and antierythropoietin antibodies in patients treated with recombinant erythropoietin. N Engl J Med. 2002;346:469-75.

15. McKoy JM, Stonecash RE, Cournoyer D, et al. Epoetin-associated pure red cell aplasia: past, present, and future considerations. Transfusion. 2008;48: 1754-62.

16. Rainville N, Jachimowicz E, Wojchowski DM. Targeting EPO and EPO receptor pathways in anemia and dysregulated erythropoiesis. Expert Opin Ther Targets. 2016;20:287-301.

17. Macdougall IC. New anemia therapies: translating novel strategies from bench to bedside. Am J Kidney Dis. 2012;59:444-51.

18. Chang HH, Chiang YW, Lin TK, et al. Erythrocytic mobilization enhanced by the granulocyte colony-stimulating factor is associated with reduced anthrax-lethal-toxin-induced mortality in mice. PLoS One. 2014;9:e111149.

19. Kau JH, Lin CG, Huang HH, et al. Calyculin A sensitive protein phosphatase is required for Bacillus anthracis lethal toxin induced cytotoxicity. Curr Microbiol. 2002:44:106-11.

20. Huang $\mathrm{YH}, \mathrm{Chin} \mathrm{CC}, \mathrm{Ho} \mathrm{HN}$, et al. Pluripotency of mouse spermatogonial stem cells maintained by IGF-1- dependent pathway. FASEB J. 2009;23: 2076-87.

21. Chang HH, Wang TP, Chen PK, et al. Erythropoiesis suppression is associated with anthrax lethal toxin-mediated pathogenic progression. PLoS One. 2013; 8:e71718.

22. Li Q, Kim Y, Namm J, et al. RNA-selective, live cell imaging probes for studying nuclear structure and function. Chem Biol. 2006;13:615-23.

23. Socolovsky M, Nam H. Fleming MD, et al. Ineffective erythropoiesis in Stat5a(-/-)5b(-/-) mice due to decreased survival of early erythroblasts. Blood. 2001:98:3261-73.

24. Metcalf D, Nicola NA. Proliferative effects of purified granulocyte colonystimulating factor (G-CSF) on normal mouse hemopoietic cells. J Cell Physiol. 1983;116:198-206.

25. Mebius RE, Kraal G. Structure and function of the spleen. Nat Rev Immunol. 2005:5:606-16.
26. Lee $\mathrm{E}$, Choi HS, Hwang $\mathrm{JH}$, et al. The RNA in reticulocytes is not just debris: it is necessary for the final stages of erythrocyte formation. Blood Cells Mol Dis. 2014;53:1-10.

27. Liu SP, Lee SD, Lee HT, et al. Granulocyte colony-stimulating factor activating HIF-1alpha acts synergistically with erythropoietin to promote tissue plasticity. PLoS One. 2010;5:e10093.

28. Moayeri M, Haines D, Young HA, et al. Bacillus anthracis lethal toxin induces TNF-alpha-independent hypoxia-mediated toxicity in mice. J Clin Invest. 2003:112:670-82.

29. Norman KE, Scheding C, Kunkel EJ, et al. Peptides derived from the lectin domain of selectin adhesion molecules inhibit leukocyte rolling in vivo. Microcirculation. 1996;3:29-38.

30. Suarez-Alvarez B, Lopez-Vazquez A, Lopez-Larrea C. Mobilization and homing of hematopoietic stem cells. Adv Exp Med Biol. 2012;741:152-70.

31. Miszti-Blasius K, Felszeghy S, Kiss C, et al. P-selectin glycoprotein ligand-1 deficiency augments G-CSF induced myeloid cell mobilization. Naunyn Schmiedebergs Arch Pharmacol. 2014:387:109-18.

32. Nomura S, Inami N, Kanazawa S, et al. Elevation of platelet activation markers and chemokines during peripheral blood stem cell harvest with G-CSF. Stem Cells. 2004:22:696-703.

33. Ohsaka A, Saionji K, Igari J. Granulocyte colony-stimulating factor administration increases serum concentrations of soluble selectins. Br J Haematol. 1998;100:66-9.

34. Haferlach T. Molecular genetics in myelodysplastic syndromes. Leuk Res. 2012;36:1459-62.

35. Raza A, Galili N. The genetic basis of phenotypic heterogeneity in myelodysplastic syndromes. Nat Rev Cancer. 2012;12:849-59.

36. Platzbecker U, Hofbauer LC, Ehninger G, et al. The clinical, quality of life, and economic consequences of chronic anemia and transfusion support in patients with myelodysplastic syndromes. Leuk Res. 2012;36:525-36.

37. Moyo V, Lefebvre P, Duh MS, et al. Erythropoiesis-stimulating agents in the treatment of anemia in myelodysplastic syndromes: a meta-analysis. Ann Hematol. 2008:87:527-36.

38. Casadevall N, Durieux P, Dubois S, et al. Health, economic, and quality-of-life effects of erythropoietin and granulocyte colony-stimulating factor for the treatment of myelodysplastic syndromes: a randomized, controlled trial. Blood. 2004:104:321-7.

39. Negrin RS, Stein R, Vardiman J, et al. Treatment of the anemia of myelodysplastic syndromes using recombinant human granulocyte colonystimulating factor in combination with erythropoietin. Blood. 1993;82:737-43.

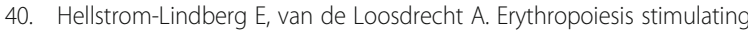
agents and other growth factors in low-risk MDS. Best Pract Res Clin Haematol. 2013;26:401-10.

41. Nikpour M, Pellagatti A, Liu A, et al. Gene expression profiling of erythroblasts from refractory anaemia with ring sideroblasts (RARS) and effects of G-CSF. Br J Haematol. 2010;149:844-54.

42. Tehranchi R, Fadeel B, Schmidt-Mende J, et al. Antiapoptotic role of growth factors in the myelodysplastic syndromes: concordance between in vitro and in vivo observations. Clin Cancer Res. 2005;11:6291-9.

43. Newman K, Maness-Harris L, El-Hemaidi I, et al. Revisiting use of growth factors in myelodysplastic syndromes. Asian Pac J Cancer Prev. 2012;13: 1081-91.

44. Singh P, Hoggatt J, Kamocka MM, et al. Neuropeptide $Y$ regulates a vascular gateway for hematopoietic stem and progenitor cells. J Clin Invest. 2017; 127(12):4527-40.

45. Itkin T, Gomez-Salinero JM, Rafii S. Open the gates: vascular neurocrine signaling mobilizes hematopoietic stem and progenitor cells. J Clin Invest. 2017;127:4231-4.

46. Bessho M, Hirashima K, Asano S, et al. Treatment of the anemia of aplastic anemia patients with recombinant human erythropoietin in combination with granulocyte colony-stimulating factor: a multicenter randomized controlled study. Multicenter Study Group. Eur J Haematol. 1997;58:265-72.

47. Miles SA, Mitsuyasu RT, Lee K, et al. Recombinant human granulocyte colony-stimulating factor increases circulating burst forming unit-erythron and red blood cell production in patients with severe human immunodeficiency virus infection. Blood. 1990;75:2137-42. 